\title{
Multiplicity Results via Topological Degree for Impulsive Boundary Value Problems under Non-Well-Ordered Upper and Lower Solution Conditions
}

\author{
Xu Xian, ${ }^{1}$ Donal O'Regan, ${ }^{2}$ and R. P. Agarwal ${ }^{3}$ \\ ${ }^{1}$ Department of Mathematics, Xuzhou Normal University, Xuzhou, Jiangsu 221116, China \\ ${ }^{2}$ Department of Mathematics, National University of Ireland, Galway, Ireland \\ ${ }^{3}$ Department of Mathematical Science, Florida Institute of Technology, Melbourne, FL 32901, USA
}

Correspondence should be addressed to R. P. Agarwal, agarwal@fit.edu

Received 14 April 2008; Accepted 26 August 2008

Recommended by R. P. Gilbert

Some multiplicity results for solutions of an impulsive boundary value problem are obtained under the condition of non-well-ordered upper and lower solutions. The main ideas of this paper are to associate a Leray-Schauder degree with the lower or upper solution.

Copyright (C) $2008 \mathrm{Xu}$ Xian et al. This is an open access article distributed under the Creative Commons Attribution License, which permits unrestricted use, distribution, and reproduction in any medium, provided the original work is properly cited.

\section{Introduction}

In this paper, we study multiplicity of solutions of the impulsive boundary value problem

$$
\begin{gathered}
y^{\prime \prime}+f\left(t, y(t), y^{\prime}(t)\right)=0, \quad t \neq t_{k}, \\
\left.\Delta y\right|_{t=t_{k}}=I_{k}\left(y\left(t_{k}\right)\right), \quad k=1,2, \ldots, m, \\
\left.\Delta y^{\prime}\right|_{t=t_{k}}=\bar{I}_{k}\left(y\left(t_{k}\right)\right), \quad k=1,2, \ldots, m, \\
y(0)=0=y(1)-\alpha y(\eta),
\end{gathered}
$$

where $f \in C\left(J \times \mathbb{R}^{2}, \mathbb{R}\right), J=[0,1], I_{k}, \bar{I}_{k} \in C(\mathbb{R}, \mathbb{R}), k=1,2, \ldots, m,\left.\Delta y\right|_{t=t_{k}}=y\left(t_{k}^{+}\right)-y\left(t_{k}^{-}\right)$, $\left.\Delta y^{\prime}\right|_{t=t_{k}}=y^{\prime}\left(t_{k}^{+}\right)-y^{\prime}\left(t_{k}^{-}\right), 0=t_{0}<t_{1}<\cdots<t_{m}<t_{m+1}=1, \alpha \in[0,1)$.

Impulsive differential equations arise naturally in a wide variety of applications, such as spacecraft control, inspection processes in operations research, drug administration, and threshold theory in biology. In the past twenty years, a significant development in the 
theory of impulsive differential equations was seen. Many authors have studied impulsive differential equations using a variety of methods (see [1-5] and the references therein).

The purpose of this paper is to study the multiplicity of solutions of the impulsive boundary value problems (1.1) by the method of upper and lower solutions. The method of lower and upper solutions has a very long history. Some of the ideas can be traced back to Picard [6]. This method deals mainly with existence results for various boundary value problems. For an overview of this method for ordinary differential equations, the reader is referred to [7]. Usually, when one uses the method of upper and lower solutions to study the existence and multiplicity of solutions of impulsive differential equations, one assumes that the upper solution is larger than the lower solution, that is, the condition that upper and lower solutions are well ordered. For example, Guo [1] studied the PBVP for second-order integrodifferential equations of mixed type in real Banach space $E$ :

$$
\begin{gathered}
-u^{\prime \prime}=f(t, u, T u, S u) \quad \forall t \in[0,2 \pi], t \neq t_{i}, \\
\left.\Delta u\right|_{t=t_{i}}=L_{i} u^{\prime}\left(t_{i}\right), \\
\left.\Delta u^{\prime}\right|_{t=t_{i}}=L_{i}^{*} u\left(t_{i}\right), \quad i=1,2, \ldots, m, \\
u(0)=u(2 \pi), \quad u^{\prime}(0)=u^{\prime}(2 \pi),
\end{gathered}
$$

where $f \in C([0,2 \pi] \times E \times E \times E, E), T$ and $S: E \mapsto E$ are two linear operators, $0<t_{1}<t_{2}<\cdots<$ $t_{m}<1, L_{i}, L_{i}^{*}(i=1,2, \ldots, m)$ are constants. In [1] Guo first obtained a comparison result, and then, by establishing two increasing and decreasing sequences, he proved an existence result for maximal and minimal solutions of the PBVP (1.2) in the ordered interval defined by the lower and upper solutions.

However, to the best of our knowledge, only in the last few years, it was shown that existence and multiplicity for impulsive differential equation under the condition that the upper solution is not larger than the lower solution, that is, the condition of non-well-ordered upper and lower solutions. In [8], Rachůnková and Tvrdý studied the existence of solutions of the nonlinear impulsive periodic boundary value problem

$$
\begin{gathered}
u^{\prime \prime}=f\left(t, u, u^{\prime}\right), \quad t \neq t_{i} \\
u\left(t_{i}^{+}\right)=2\left(u\left(t_{i}\right)\right), \quad i=1,2, \ldots, m, \\
u^{\prime}\left(t_{i}^{+}\right)=\mathcal{M}_{i}\left(u^{\prime}\left(t_{i}\right)\right), \quad i=1,2, \ldots, m, \\
u(0)=u(T), \quad u^{\prime}(0)=u^{\prime}(T),
\end{gathered}
$$

where $f \in C\left([0, T] \times \mathbb{R}^{2}\right), \partial_{i}, \mathcal{M}_{i} \in C(\mathbb{R})$. Using Leray-Schauder degree, the authors of [8] showed some existence results for (1.3) under the non-well-ordered upper and lower solutions condition. For other results related to non-well-ordered upper and lower solutions, the reader is referred to $[7,9-14]$. Also, here we mention the main results of a very recent paper [15]. In that paper, we studied the second-order three-point boundary value problem

$$
\begin{aligned}
& y^{\prime \prime}(t)+f(t, y)=0, \quad 0 \leq t \leq 1, \\
& y(0)=0, \quad y(1)-\alpha y(\eta)=0
\end{aligned}
$$

where $0<\eta<1,0<\alpha<1, f \in C([0,1] \times \mathbb{R}, \mathbb{R})$. In [15], we made the following assumption. 


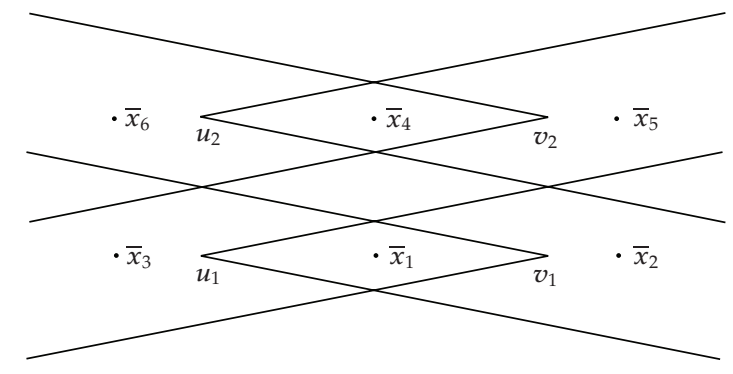

Figure 1: The positions of $u_{1}, u_{2}, v_{1}, v_{2}$ and six solutions $\bar{x}_{1}, \bar{x}_{2}, \ldots, \bar{x}_{6}$ in Theorem 1.1.

$\left(A_{0}\right)$ There exists $M>0$ such that

$$
f\left(t, x_{2}\right)-f\left(t, x_{1}\right) \geq-M\left(x_{2}-x_{1}\right), \quad t \in[0,1], x_{2} \geq x_{1} .
$$

Let the function $e$ be $e=e(t)=t$ for $t \in[0,1]$. In [15], we proved the following theorem (see, [15, Theorem 3.4]).

Theorem 1.1. Suppose that $\left(A_{0}\right)$ holds, $u_{1}$ and $u_{2}$ are two strict lower solutions of (1.4), $v_{1}$ and $v_{2}$ are two strict upper solutions of (1.4), and $u_{1}<v_{1}, u_{2}<v_{2}, u_{2} \leq v_{1}, u_{1} \not v_{2}$. Moreover, assume

$$
-\varsigma_{0} e \leq u_{2}-u_{1} \leq \varsigma_{0} e, \quad-\varsigma_{0} e \leq v_{2}-v_{1} \leq \varsigma_{0} e
$$

for some $s_{0}>0$. Then the three-point boundary value problem (1.4) has at least six solutions $\bar{x}_{1}, \bar{x}_{2}, \ldots, \bar{x}_{6}$.

Theorem 1.1 establishes the existence of at least six solutions of the three-point boundary value problem (1.4) only under the condition of two pairs of strict lower and upper solutions. The positions of $u_{1}, u_{2}, v_{1}, v_{2}$ and six solutions $\bar{x}_{1}, \bar{x}_{2}, \ldots, \bar{x}_{6}$ in Theorem 1.1 can be illustrated roughly by Figure 1 .

In some sense, we can say that these two pairs of lower and upper solutions are parallel to each other. The position of these two pairs of lower and upper solutions is sharply different from that of the lower and upper solutions of the main results in $[14,16,17]$. The technique to prove our main results of [15] is to use the fixed-point index of some increasing operator with respect to some closed convex sets, which are translations of some special cones (see $Q_{c}$, $Q_{\bar{c}}$ of [15]).

This paper is a continuation of the paper [15]. The aim of this paper is to study the multiplicity of solutions of the impulsive boundary value problem (1.1) under the conditions of non-well-ordered upper and lower solutions. In this paper, we will permit the presence of impulses and the first derivative. The main ideas of this paper are to associate a LeraySchauder degree with the lower or upper solution. We will give some multiplicity results for at least eight solutions. To obtain this multiplicity result, an additional pair of lower and upper solutions is needed, that is, we will employ a condition of three pairs of lower and upper solutions. The position of these three pairs of lower and upper solutions will be illustrated in Remark 2.16. 


\section{Results for at least eight solutions}

Let $J^{\prime}=J \backslash\left\{t_{1}, t_{2}, \ldots, t_{m}\right\}, \mathrm{PC}[J, \mathbb{R}]=\{x \mid x$ is a map from $J$ into $\mathbb{R}$ such that $x(t)$ is continuous at $t \neq t_{k}$, left continuous at $t=t_{k}$ and its right-hand limit $x\left(t_{k}^{+}\right)$at $t=t_{k}$ exits $\}$, and $\operatorname{PC}^{1}[J, \mathbb{R}]=$ $\left\{x \mid x\right.$ is a map from $J$ into $\mathbb{R}$ such that $x(t)$ and $x^{\prime}(t)$ are continuous at $t \neq t_{k}$, left continuous at $t=t_{k}$ and their right-hand limits $x\left(t_{k}^{+}\right)$and $x^{\prime}\left(t_{k}^{+}\right)$at $t=t_{k}$ exits $\}$. For each $x \in \operatorname{PC}^{1}[J, \mathbb{R}]$, let

$$
\|x\|_{\mathrm{PC}^{1}}=\max \left\{\|x\|_{\mathrm{PC}},\left\|x^{\prime}\right\|_{\mathrm{PC}}\right\},
$$

where $\|x\|_{\mathrm{PC}}=\sup _{t \in J}|x(t)|$ and $\left\|x^{\prime}\right\|_{\mathrm{PC}}=\sup _{t \in J}\left|x^{\prime}(t)\right|$. Then, $\mathrm{PC}^{1}[J, \mathbb{R}]$ is a real Banach space with the norm $\|\cdot\|_{\mathrm{PC}^{1}}$. The function $x \in \operatorname{PC}^{1}[J, \mathbb{R}] \cap C^{2}\left[J^{\prime}, \mathbb{R}\right]$ is called a solution of the boundary value problem (1.1) if it satisfies all the equalities of (1.1).

Now, for convenience, we make the following assumptions.

$\left(H_{0}\right) 0=t_{0}<t_{1}<\cdots<t_{m}<\eta<t_{m+1}=1, \alpha \in[0,1)$.

$\left(H_{1}\right) I_{k}(k=1,2, \ldots, m)$ is increasing on $\mathbb{R}$.

Let $x, y \in \mathrm{PC}[J, \mathbb{R}]$. Now, we define the ordering $\prec$ by

$$
x<y \quad \text { iff } x(t)<y(t) \forall t \in J, \quad x\left(t_{k}^{+}\right)<y\left(t_{k}^{+}\right) \quad \text { for each } k=1,2, \ldots, m .
$$

Definition 2.1. The function $u \in \mathrm{PC}^{1}[J, \mathbb{R}] \cap C^{2}\left[J^{\prime}, \mathbb{R}\right]$ is called a strict lower solution of (1.1) if

$$
\begin{gathered}
u^{\prime \prime}(t)+f\left(t, u(t), u^{\prime}(t)\right)>0, \quad t \neq t_{k} \\
u(0)<0, \quad u(1)-\alpha u(\eta)<0
\end{gathered}
$$

whenever $I_{i_{0}}(x) \neq 0$ or $\bar{I}_{j_{0}}(x) \neq 0$ for some $i_{0}, j_{0} \in\{1,2, \ldots, m\}$ and some $x \in \mathbb{R}$

$$
\begin{array}{cc}
\left.\Delta u\right|_{t=t_{k}}=I_{k}\left(u\left(t_{k}\right)\right), & k=1,2, \ldots, m, \\
\left.\Delta u^{\prime}\right|_{t=t_{k}}>\bar{I}_{k}\left(u\left(t_{k}\right)\right), & k=1,2, \ldots, m,
\end{array}
$$

whenever $I_{k}(x)=\bar{I}_{k}(x)=0$ for each $x \in \mathbb{R}$ and $k \in\{1,2, \ldots, m\},\left.\Delta u\right|_{t=t_{k}}=\left.\Delta u^{\prime}\right|_{t=t_{k}}=0$ for each $k \in\{1,2, \ldots, m\}$.

The function $v \in \operatorname{PC}^{1}[J, \mathbb{R}] \cap C^{2}\left[J^{\prime}, R\right]$ is called a strict upper solution of (1.1) if

$$
\begin{gathered}
v^{\prime \prime}(t)+f\left(t, v(t), v^{\prime}(t)\right)<0, \quad t \neq t_{k} \\
v(0)>0, \quad v(1)-\alpha v(\eta)>0,
\end{gathered}
$$

whenever $I_{i_{0}}(x) \neq 0$ or $\bar{I}_{j_{0}}(x) \neq 0$ for some $i_{0}, j_{0} \in\{1,2, \ldots, m\}$ and some $x \in \mathbb{R}$

$$
\begin{aligned}
\left.\Delta v\right|_{t=t_{k}} & =I_{k}\left(v\left(t_{k}\right)\right), \quad k=1,2, \ldots, m, \\
\left.\Delta v^{\prime}\right|_{t=t_{k}}<\bar{I}_{k}\left(v\left(t_{k}\right)\right), & k=1,2, \ldots, m,
\end{aligned}
$$

and whenever $I_{k}(x)=\bar{I}_{k}(x)=0$ for each $x \in \mathbb{R}$ and $k \in\{1,2, \ldots, m\},\left.\Delta v\right|_{t=t_{k}}=\left.\Delta v^{\prime}\right|_{t=t_{k}}=0$ for each $k \in\{1,2, \ldots, m\}$. 
Definition 2.2. Let $u(t), v(t) \in \operatorname{PC}^{1}[J, \mathbb{R}] \cap C^{2}\left[J^{\prime}, \mathbb{R}\right], u(t) \leq v(t)$ for all $t \in J$. We say that $f$ satisfies Nagumo condition with respect to $[u, v]$ if there exists function $\phi \in C([0, \infty),(0, \infty))$ such that

$$
\begin{gathered}
|f(t, x, y)| \leq \phi(|y|), \quad \forall(t, x, y) \in J \times[u(t), v(t)] \times \mathbb{R}, \\
\int_{0}^{\infty} \frac{s}{\phi(s)} d s=\infty .
\end{gathered}
$$

Definition 2.3. Let $r_{1}(t), r_{2}(t) \cdots r_{n}(t)$ be strict upper solutions of (1.1) and $r(t)=$ $\min \left\{r_{1}(t), r_{2}(t), \ldots r_{n}(t)\right\}$ for each $t \in J$. Then, we say the upper solutions $r_{1}(t), r_{2}(t), \ldots r_{n}(t)$ are well ordered if for each $k \in\{1,2, \ldots, m\}$, there exist $i_{0}, j_{0} \in\{1,2, \ldots, n\}$ and $\delta_{0}>0$ small enough such that

$$
r(t)= \begin{cases}r_{i_{0}}(t), & t \in\left(t_{k}-\delta_{0}, t_{k}\right], \\ r_{j_{0}}(t), & t \in\left(t_{k}, t_{k}+\delta_{0}\right] .\end{cases}
$$

Definition 2.4. Let $l_{1}(t), l_{2}(t), \ldots l_{n}(t)$ be strict lower solutions of (1.1) and $l(t)=$ $\max \left\{l_{1}(t), l_{2}(t), \ldots l_{n}(t)\right\}$ for each $t \in J$. Then, we say the lower solutions $l_{1}(t), l_{2}(t), \ldots l_{n}(t)$ are well ordered if for each $k \in\{1,2, \ldots, m\}$, there exist $i_{0}, j_{0} \in\{1,2, \ldots, n\}$ and $\delta_{0}>0$ small enough such that

$$
l(t)= \begin{cases}l_{i_{0}}(t), & t \in\left(t_{k}-\delta_{0}, t_{k}\right], \\ l_{j_{0}}(t), & t \in\left(t_{k}, t_{k}+\delta_{0}\right] .\end{cases}
$$

From [18, Lemma 5.4.1], we have the following lemma.

Lemma 2.5. $H \subset \mathrm{PC}^{1}[J, \mathbb{R}]$ is a relative compact set if and only if for all $x \in H, x(t)$ and $x^{\prime}(t)$ are uniformly bounded on $J$ and equicontinuous on each $J_{k}(k=1,2, \ldots, m)$, where $J_{1}=\left[0, t_{1}\right], J_{i}=$ $\left(t_{i-1}, t_{i}\right], i=2,3, \ldots, m+1$.

The following lemma can be easily proved.

Lemma 2.6. Suppose that $x \in \operatorname{PC}^{1}[J, \mathbb{R}] \cap C^{2}\left[J^{\prime}, \mathbb{R}\right]$ satisfies

$$
-x^{\prime \prime}(t)=f\left(t, x(t), x^{\prime}(t)\right), \quad t \neq t_{k}(k=1,2, \ldots, m) .
$$

Then

$$
\begin{aligned}
x^{\prime}(t)= & x^{\prime}(0)-\int_{0}^{t} f\left(s, x(s), x^{\prime}(s)\right) d s+\sum_{0<t_{k}<t}\left[x^{\prime}\left(t_{k}^{+}\right)-x^{\prime}\left(t_{k}\right)\right] \quad \forall t \in J, \\
x(t)= & x(0)+x^{\prime}(0) t-\int_{0}^{t}(t-s) f\left(s, x(s), x^{\prime}(s)\right) d s+\sum_{0<t_{k}<t}\left[x\left(t_{k}^{+}\right)-x\left(t_{k}\right)\right] \\
& +\sum_{0<t_{k}<t}\left[x^{\prime}\left(t_{k}^{+}\right)-x^{\prime}\left(t_{k}\right)\right]\left(t-t_{k}\right) \quad \forall t \in J .
\end{aligned}
$$


Lemma 2.7. Let $g \in \mathrm{PC}[J, \mathbb{R}]$ and $a_{k}, b_{k} \in \mathbb{R}(k=0,1,2, \ldots, m)$. Then, $x \in \operatorname{PC}^{1}[J, \mathbb{R}] \cap C^{2}\left[J^{\prime}, \mathbb{R}\right]$ is a solution of

$$
\begin{gathered}
-x^{\prime \prime}(t)=g(t), \quad t \neq t_{k}, k=1,2, \ldots, m, \\
\left.\Delta x\right|_{t=t_{k}}=a_{k}, \quad k=1,2, \ldots, m, \\
\left.\Delta x^{\prime}\right|_{t=t_{k}}=b_{k}, \quad k=1,2, \ldots, m, \\
x(0)=a_{0}, \quad x(1)-\alpha x(\eta)=b_{0}
\end{gathered}
$$

if and only if $x \in \mathrm{PC}[J, \mathbb{R}]$ satisfies

$$
\begin{aligned}
x(t)= & a_{0}\left[1-\frac{1-\alpha}{1-\alpha \eta} t\right]+\frac{b_{0} t}{1-\alpha \eta}+\frac{t}{1-\alpha \eta} \int_{0}^{1}(1-s) g(s) d s-\frac{\alpha t}{1-\alpha \eta} \int_{0}^{\eta}(\eta-s) g(s) d s \\
& -\frac{t}{1-\alpha \eta} \sum_{k=1}^{m}\left\{(1-\alpha) a_{k}+\left[1-t_{k}-\alpha\left(\eta-t_{k}\right)\right] b_{k}\right\}-\int_{0}^{t}(t-s) g(s) d s \\
& +\sum_{0<t_{k}<t}\left[a_{k}+b_{k}\left(t-t_{k}\right)\right], \quad t \in J .
\end{aligned}
$$

Proof. Let $x \in \mathrm{PC}^{1}[J, \mathbb{R}] \cap C^{2}\left[J^{\prime}, \mathbb{R}\right]$ be a solution of (2.12). From Lemma 2.6, we have

$$
\begin{aligned}
x(t) & =x(0)+x^{\prime}(0) t-\int_{0}^{t}(t-s) g(s) d s+\left.\sum_{0<t_{k}<t} \Delta x\right|_{t=t_{k}}+\left.\sum_{0<t_{k}<t} \Delta x^{\prime}\right|_{t=t_{k}}\left(t-t_{k}\right) \\
& =a_{0}+x^{\prime}(0) t-\int_{0}^{t}(t-s) g(s) d s+\sum_{0<t_{k}<t} a_{k}+\sum_{0<t_{k}<t} b_{k}\left(t-t_{k}\right) .
\end{aligned}
$$

Thus,

$$
\begin{aligned}
& x(1)=a_{0}+x^{\prime}(0)-\int_{0}^{1}(1-s) g(s) d s+\sum_{k=1}^{m} a_{k}+\sum_{k=1}^{m} b_{k}\left(1-t_{k}\right), \\
& x(\eta)=a_{0}+x^{\prime}(0) \eta-\int_{0}^{\eta}(\eta-s) g(s) d s+\sum_{k=1}^{m} a_{k}+\sum_{k=1}^{m} b_{k}\left(\eta-t_{k}\right) .
\end{aligned}
$$

Using the boundary value condition $x(1)-\alpha x(\eta)=b_{0}$, we have

$$
\begin{aligned}
x^{\prime}(0)= & \frac{1}{1-\alpha \eta} b_{0}-\frac{1-\alpha}{1-\alpha \eta} a_{0}+\frac{1}{1-\alpha \eta} \int_{0}^{1}(1-s) g(s) d s-\frac{\alpha}{1-\alpha \eta} \int_{0}^{\eta}(\eta-s) g(s) d s \\
& -\frac{1-\alpha}{1-\alpha \eta} \sum_{k=1}^{m} a_{k}-\frac{1}{1-\alpha \eta} \sum_{k=1}^{m} b_{k}\left(1-t_{k}\right)+\frac{\alpha}{1-\alpha \eta} \sum_{k=1}^{m} b_{k}\left(\eta-t_{k}\right) .
\end{aligned}
$$

The equality (2.13) now follows from (2.14) and (2.16). 
On the other hand, if $x \in \mathrm{PC}[J, \mathbb{R}]$ satisfies (2.13), by direct computation, we can easily show that $x$ satisfies (2.12). The proof is complete.

Let us define the operator $A: \mathrm{PC}^{1}[J, \mathbb{R}] \mapsto \mathrm{PC}^{1}[J, \mathbb{R}]$ by

$$
\begin{aligned}
(A x)(t)= & \frac{t}{1-\alpha \eta} \int_{0}^{1}(1-s) f\left(s, x(s), x^{\prime}(s)\right) d s-\frac{\alpha t}{1-\alpha \eta} \int_{0}^{\eta}(\eta-s) f\left(s, x(s), x^{\prime}(s)\right) d s \\
& -\int_{0}^{t}(t-s) f\left(s, x(s), x^{\prime}(s)\right) d s+\sum_{0<t_{k}<t}\left[I_{k}\left(x\left(t_{k}\right)\right)+\bar{I}_{k}\left(x\left(t_{k}\right)\right)\left(t-t_{k}\right)\right] \\
& -\frac{t}{1-\alpha \eta} \sum_{k=1}^{m}\left\{(1-\alpha) I_{k}\left(x\left(t_{k}\right)\right)+\left[1-t_{k}-\alpha\left(\eta-t_{k}\right)\right] \bar{I}_{k}\left(x\left(t_{k}\right)\right)\right\} .
\end{aligned}
$$

From Lemma 2.5, $A: \mathrm{PC}^{1}[J, \mathbb{R}] \mapsto \mathrm{PC}^{1}[J, \mathbb{R}]$ is a completely continuous operator.

Theorem 2.8. Suppose that $\left(H_{0}\right)$ and $\left(H_{1}\right)$ hold. Let $\alpha_{i}, \beta_{i}(i=1,2, \ldots, n)$ be $n$ pairs of strict lower and upper solution, and

$$
\begin{aligned}
& \bar{\alpha}(t)=\max \left\{\alpha_{1}(t), \alpha_{2}(t), \ldots, \alpha_{n}(t)\right\}, \quad t \in J, \\
& \bar{\beta}(t)=\min \left\{\beta_{1}(t), \beta_{2}(t), \ldots, \beta_{n}(t)\right\}, \quad t \in J .
\end{aligned}
$$

Suppose that $\alpha_{i} \prec \beta_{i}(i=1,2, \ldots, n), \bar{\alpha} \prec \bar{\beta}$, $f$ satisfies Nagumo condition with respect to $\left[\alpha_{1}, \beta_{1}\right]$. Moreover, the strict lower solutions $\alpha_{1}, \alpha_{2}, \ldots, \alpha_{n}$ and the strict upper solutions $\beta_{1}, \beta_{2}, \ldots, \beta_{n}$ are well ordered whenever $I_{i_{0}}(x) \neq 0$ or $\bar{I}_{j_{0}}(x) \neq 0$ for some $i_{0}, j_{0} \in\{1,2, \ldots, m\}$ and some $x \in \mathbb{R}$. Then, there exist $R_{0}>0$ and $L_{0}>0$ sufficiently large such that for each $R \geq R_{0}$ and $L>L_{0}$

$$
\operatorname{deg}(I-A, \Omega, \theta)=1,
$$

where

$$
\begin{gathered}
\Omega=\left\{x \in B(\theta, R) \mid \bar{\alpha} \prec x<\bar{\beta},-L \prec x^{\prime}<L\right\}, \\
B(\theta, R)=\left\{x \in \operatorname{PC}^{1}[J, R] \mid\|x\|_{\mathrm{PC}^{1}}<R\right\} .
\end{gathered}
$$

Proof. We only prove the case when $I_{i_{0}}(x) \neq 0$ or $\bar{I}_{j_{0}}(x) \neq 0$ for some $i_{0}, j_{0} \in\{1,2, \ldots, m\}$ and some $x \in \mathbb{R}$. The conclusion is achieved in four steps.

Step 1. Since $f$ satisfies Nagumo condition with respect to $\left[\alpha_{1}, \beta_{1}\right]$, then there exists $\phi \in$ $C([0, \infty),(0, \infty))$ such that

$$
\begin{gathered}
|f(t, x, y)| \leq \phi(|y|), \quad(t, x, y) \in J \times[\alpha(t), \beta(t)] \times \mathbb{R}, \\
\int_{0}^{\infty} \frac{s}{\phi(s)} d s=\infty .
\end{gathered}
$$


Let $\mu_{0}=\min _{1 \leq k \leq m+1}\left(t_{k}-t_{k-1}\right)$. Take $\lambda>0$ such that

$$
\lambda>\frac{\max _{1 \leq i \leq n} \sup _{t \in J} \beta_{i}(t)-\min _{1 \leq i \leq n} \inf _{t \in J} \alpha_{i}(t)}{\mu_{0}},
$$

and $N>0$ such that

$$
\int_{\lambda}^{N} \frac{s}{\phi(s)} d s>2 \lambda
$$

Let $L_{0}=\max \left\{N, 2 \lambda, \max _{1 \leq i \leq n}\left\|\alpha_{i}^{\prime}\right\|_{\mathrm{PC}}, \max _{1 \leq i \leq n}\left\|\beta_{i}^{\prime}\right\|_{\mathrm{PC}}\right\}$. Define the functions $g, h: J \times \mathbb{R}^{2} \mapsto \mathbb{R}$ by

$$
\begin{aligned}
& g(t, x, y)= \begin{cases}f(t, x, L), & y>L, \\
f(t, x, y), & -L \leq y \leq L, \\
f(t, x,-L), & y<-L,\end{cases} \\
& h(t, x, y)= \begin{cases}g(t, \bar{\beta}(t), y), & x>\bar{\beta}(t), \\
g(t, x, y), & \bar{\alpha}(t) \leq x \leq \bar{\beta}(t), \\
g(t, \bar{\alpha}(t), y), & x<\bar{\alpha}(t) .\end{cases}
\end{aligned}
$$

For each $k \in\{1,2, \ldots, m\}$, let us define the functions $J_{k}, \bar{J}_{k}: \mathbb{R} \mapsto \mathbb{R}$ by

$$
\begin{aligned}
& J_{k}(x)= \begin{cases}I_{k}\left(\bar{\beta}\left(t_{k}\right)\right), & x>\bar{\beta}\left(t_{k}\right), \\
I_{k}(x), & \bar{\alpha}\left(t_{k}\right) \leq x \leq \bar{\beta}\left(t_{k}\right), \\
I_{k}\left(\bar{\alpha}\left(t_{k}\right)\right), & x<\bar{\alpha}\left(t_{k}\right),\end{cases} \\
& \bar{J}_{k}(x)= \begin{cases}\bar{I}_{k}\left(\bar{\beta}\left(t_{k}\right)\right), & x>\bar{\beta}\left(t_{k}\right), \\
\bar{I}_{k}(x), & \bar{\alpha}\left(t_{k}\right) \leq x \leq \bar{\beta}\left(t_{k}\right), \\
\bar{I}_{k}\left(\bar{\alpha}\left(t_{k}\right)\right), & x<\bar{\alpha}\left(t_{k}\right) .\end{cases}
\end{aligned}
$$

It is easy to see that there exists $M_{1}>0$ such that

$$
\begin{gathered}
|h(t, x, y)| \leq M_{1}, \quad(t, x, y) \in J \times \mathbb{R}^{2}, \\
\left|J_{k}(x)\right| \leq M_{1}, \quad\left|\bar{J}_{k}(x)\right| \leq M_{1}, \quad x \in \mathbb{R}, k=1,2, \ldots, m .
\end{gathered}
$$


Xu Xian et al.

Let us define the operator $A^{*}: \mathrm{PC}^{1}[J, \mathbb{R}] \mapsto \mathrm{PC}^{1}[J, \mathbb{R}]$ by

$$
\begin{aligned}
\left(A^{*} x\right)(t)= & \frac{t}{1-\alpha \eta} \int_{0}^{1}(1-s) h\left(s, x(s), x^{\prime}(s)\right) d s-\frac{\alpha t}{1-\alpha \eta} \int_{0}^{\eta}(\eta-s) h\left(s, x(s), x^{\prime}(s)\right) d s \\
& -\int_{0}^{t}(t-s) h\left(s, x(s), x^{\prime}(s)\right) d s+\sum_{0<t_{k}<t}\left[J_{k}\left(x\left(t_{k}\right)\right)+\left(t-t_{k}\right) \bar{J}_{k}\left(x\left(t_{k}\right)\right)\right] \\
& -\frac{t}{1-\alpha \eta} \sum_{k=1}^{m}\left\{(1-\alpha) J_{k}\left(x\left(t_{k}\right)\right)+\left[\left(1-t_{k}\right)-\alpha\left(\eta-t_{k}\right)\right] \bar{J}_{k}\left(x\left(t_{k}\right)\right)\right\} .
\end{aligned}
$$

By (2.26), we have

$$
\begin{aligned}
\left(A^{*} x\right)(t) \leq & \frac{1}{1-\alpha \eta} \int_{0}^{1}(1-s)\left|h\left(s, x(s), x^{\prime}(s)\right)\right| d s+\frac{\alpha}{1-\alpha \eta} \int_{0}^{\eta}(\eta-s)\left|h\left(s, x(s), x^{\prime}(s)\right)\right| d s \\
& +\int_{0}^{1}(1-s)\left|h\left(s, x(s), x^{\prime}(s)\right)\right| d s+\sum_{k=1}^{m}\left[\left|J_{k}\left(x\left(t_{k}\right)\right)\right|+\left(1-t_{k}\right)\left|\bar{J}_{k}\left(x\left(t_{k}\right)\right)\right|\right] \\
& +\frac{2}{1-\alpha \eta} \sum_{k=1}^{m}\left[\left|J_{k}\left(x\left(t_{k}\right)\right)\right|+\left|\bar{J}_{k}\left(x\left(t_{k}\right)\right)\right|\right] \\
\leq & \frac{M_{1}}{1-\alpha \eta}\left[\frac{1}{2}+\frac{1}{2} \alpha \eta^{2}+\frac{1}{2}+2 m+4 m\right] \\
\leq & \frac{M_{1}}{1-\alpha \eta}[3+6 m], \quad t \in J, \\
\left(A^{*} x\right)^{\prime}(t) \leq & \frac{1}{1-\alpha \eta} \int_{0}^{1}(1-s)\left|h\left(s, x(s), x^{\prime}(s)\right)\right| d s+\frac{\alpha}{1-\alpha \eta} \int_{0}^{\eta}(\eta-s)\left|h\left(s, x(s), x^{\prime}(s)\right)\right| d s \\
& +\int_{0}^{1}\left|h\left(s, x(s), x^{\prime}(s)\right)\right| d s+\sum_{k=1}^{m}\left|\bar{J}_{k}\left(x\left(t_{k}\right)\right)\right|+\frac{2}{1-\alpha \eta} \sum_{k=1}^{m}\left[\left|J_{k}\left(x\left(t_{k}\right)\right)\right|+\left|\bar{J}_{k}\left(x\left(t_{k}\right)\right)\right|\right] \\
\leq & \frac{M_{1}}{1-\alpha \eta}\left[\frac{1}{2}+\frac{1}{2} \alpha \eta^{2}+1+5 m\right] \\
\leq & \frac{M_{1}}{1-\alpha \eta}[5 m+3], \quad t \in J .
\end{aligned}
$$

From (2.28), we have $\left\|A^{*} x\right\|_{\mathrm{PC}^{1}} \leq\left(M_{1} /(1-\alpha \eta)\right)[11 m+6]$ for each $x \in \mathrm{PC}^{1}[J, \mathbb{R}]$. Let $R_{0}=$ $\left(M_{1} /(1-\alpha \eta)\right)(11 m+6)+1$. Then, $A^{*}\left(\mathrm{PC}^{1}[J, \mathbb{R}]\right) \subset B\left(\theta, R_{0}\right)$. By the properties of the LeraySchauder degree, we have

$$
\operatorname{deg}\left(I-A^{*}, B(\theta, R), \theta\right)=1 \text {. }
$$


Thus, $A^{*}$ has at least one fixed point $x_{0}$. From Lemma 2.7, $x_{0}$ satisfies

$$
\begin{gathered}
x_{0}^{\prime \prime}(t)+h\left(t, x_{0}(t), x_{0}^{\prime}(t)\right)=0, \quad t \neq t_{k}, \\
\left.\Delta x_{0}\right|_{t=t_{k}}=J_{k}\left(x_{0}\left(t_{k}\right)\right), \quad k=1,2, \ldots, m \\
\left.\Delta x_{0}^{\prime}\right|_{t=t_{k}}=\bar{J}_{k}\left(x_{0}\left(t_{k}\right)\right), \quad k=1,2, \ldots, m, \\
x_{0}(0)=0=x_{0}(1)-\alpha x_{0}(\eta),
\end{gathered}
$$

Step 2. Next, we will show that

$$
\begin{gathered}
\bar{\alpha} \prec x_{0} \prec \bar{\beta}, \\
-L \prec x_{0}^{\prime} \prec L .
\end{gathered}
$$

We first show that

$$
\bar{\alpha}(t) \leq x_{0}(t) \leq \bar{\beta}(t) \quad \forall t \in J .
$$

To begin, we show that $x_{0}(t) \leq \bar{\beta}(t)$ for all $t \in J$. Suppose not, then there exists $t^{\prime} \in J$ such that $x_{0}\left(t^{\prime}\right)>\bar{\beta}\left(t^{\prime}\right)$. Set $w(t)=x_{0}(t)-\bar{\beta}(t)$ for $t \in J$. There are a number of cases to consider.

(1) $w(0)=\sup _{t \in J} w(t)>0$, then, we have

$$
0<w(0)=x_{0}(0)-\bar{\beta}(0)=-\bar{\beta}(0)<0,
$$

which is a contradiction.

(2) $w(1)=\sup _{t \in J} w(t)>0$; assume without loss of generality that $\alpha>0$ and $\bar{\beta}(1)=$ $\beta_{i_{0}}(1)$ for some $i_{0} \in\{1,2, \ldots, n\}$, then, we have

$$
0<w(1)=x_{0}(1)-\beta_{i_{0}}(1) \leq \alpha x_{0}(\eta)-\alpha \beta_{i_{0}}(\eta) \leq \alpha x_{0}(\eta)-\alpha \bar{\beta}(\eta)=\alpha w(\eta) \leq \alpha w(1)
$$

which is a contradiction.

(3) There exist $k_{0} \in\{1,2, \ldots, m, m+1\}$ and $\tau_{0} \in\left(t_{k_{0}-1}, t_{k_{0}}\right)$ such that $w\left(\tau_{0}\right)=$ $\sup _{t \in J} w(t)>0$. Assume without loss of generality that $\bar{\beta}\left(\tau_{0}\right)=\beta_{i_{0}}\left(\tau_{0}\right)$ for some $i_{0} \in$ $\{1,2, \ldots, n\}$. We have the following two cases:

(3A) $\beta_{j}\left(\tau_{0}\right)>\beta_{i_{0}}\left(\tau_{0}\right)$ for each $j \in\{1,2, \ldots, n\}$ and $j \neq i_{0}$;

(3B) there exists $j_{0} \in\{1,2, \ldots, n\}, j_{0} \neq i_{0}$ such that $\beta_{j_{0}}\left(\tau_{0}\right)=\beta_{i_{0}}\left(\tau_{0}\right)$.

and

For case (3A), there exists $\delta_{0}>0$ small enough such that $\left[\tau_{0}-\delta_{0}, \tau_{0}+\delta_{0}\right] \subset\left(t_{k_{0}-1}, t_{k_{0}}\right)$

$$
w(t)=x_{0}(t)-\beta_{i_{0}}(t), \quad t \in\left[\tau_{0}-\delta_{0}, \tau_{0}+\delta_{0}\right]
$$


Then, $w \in C^{2}\left[\tau_{0}-\delta_{0}, \tau_{0}+\delta_{0}\right], w\left(\tau_{0}\right)$ is the maximum of $w$ on $\left[\tau_{0}-\delta_{0}, \tau_{0}+\delta_{0}\right]$. Thus, $w^{\prime}\left(\tau_{0}\right)=$ $0, w^{\prime \prime}\left(\tau_{0}\right) \leq 0$. By $(2.30)$, we have

$$
\begin{aligned}
0 & \geq w^{\prime \prime}\left(\tau_{0}\right)=x_{0}^{\prime \prime}\left(\tau_{0}\right)-\beta_{i_{0}}^{\prime \prime}\left(\tau_{0}\right)=-h\left(\tau_{0}, x_{0}\left(\tau_{0}\right), x_{0}^{\prime}\left(\tau_{0}\right)\right)-\beta_{i_{0}}^{\prime \prime}\left(\tau_{0}\right) \\
& =-f\left(\tau_{0}, \beta_{i_{0}}\left(\tau_{0}\right), \beta_{i_{0}}^{\prime}\left(\tau_{0}\right)\right)-\beta_{i_{0}}^{\prime \prime}\left(\tau_{0}\right)
\end{aligned}
$$

which is a contradiction.

For case (3B), set $w_{1}(t)=x_{0}(t)-\beta_{j_{0}}(t)$ for $t \in\left(t_{k_{0}-1}, t_{k_{0}}\right)$. For any $t^{\prime} \in\left(t_{k_{0}-1}, t_{k_{0}}\right)$, we have

$$
\begin{aligned}
w_{1}\left(\tau_{0}\right) & =x_{0}\left(\tau_{0}\right)-\beta_{j_{0}}\left(\tau_{0}\right)=x_{0}\left(\tau_{0}\right)-\beta_{i_{0}}\left(\tau_{0}\right)=w\left(\tau_{0}\right) \\
& \geq w\left(t^{\prime}\right)=x_{0}\left(t^{\prime}\right)-\bar{\beta}\left(t^{\prime}\right) \geq x_{0}\left(t^{\prime}\right)-\beta_{j_{0}}\left(t^{\prime}\right)=w_{1}\left(t^{\prime}\right) .
\end{aligned}
$$

This implies that $w_{1}\left(\tau_{0}\right)$ is a local maximum. Since $w_{1} \in C^{2}\left(t_{k_{0}-1}, t_{k_{0}}\right)$, then $w_{1}^{\prime}\left(\tau_{0}\right)=0$, $w_{1}^{\prime \prime}\left(\tau_{0}\right) \leq 0$. Therefore,

$$
0 \geq w_{1}^{\prime \prime}\left(\tau_{0}\right)=x_{0}^{\prime \prime}\left(\tau_{0}\right)-\beta_{j_{0}}^{\prime \prime}\left(\tau_{0}\right)=-f\left(\tau_{0}, \beta_{j_{0}}\left(\tau_{0}\right), \beta_{j_{0}}^{\prime}\left(\tau_{0}\right)\right)-\beta_{j_{0}}^{\prime \prime}\left(\tau_{0}\right)>0,
$$

which is a contradiction.

(4) There exists $k_{0} \in\{1,2, \ldots, m\}$ such that $w\left(t_{k_{0}}\right)=\sup _{t \in J} w(t)>0$. Without loss of generality, we may assume $w(\tau)<\sup _{t \in J} w(t)$ for each $\tau \in\left(t_{k-1}, t_{k}\right)$ and $k \in\{1,2, \ldots, m, m+$ $1\}$. (Otherwise, if there exists $\tau_{0} \in\left(t_{k_{0}-1}, t_{k_{0}}\right)$ for some $k_{0} \in\{1,2, \ldots, m, m+1\}$ such that $w\left(\tau_{0}\right)=\sup _{t \in J} w(t)$, then we can get a contradiction as in case (3)). In this case, we have the following two subcases:

(4A) there exists $i_{0} \in\{1,2, \ldots, n\}$ such that $\beta_{i_{0}}\left(t_{k_{0}}\right)<\beta_{j}\left(t_{k_{0}}\right)$ for $j=1,2, \ldots, n$ and $j \neq i_{0}$;

(4B) there exists a subset $\left\{n_{1}, n_{2}, \ldots, n_{s}\right\} \subset\{1,2, \ldots, n\}$ such that

$$
\bar{\beta}\left(t_{k_{0}}\right)=\beta_{n_{1}}\left(t_{k_{0}}\right)=\beta_{n_{2}}\left(t_{k_{0}}\right)=\cdots=\beta_{n_{s}}\left(t_{k_{0}}\right)
$$

while $\beta_{l}\left(t_{k_{0}}\right)>\bar{\beta}\left(t_{k_{0}}\right)$ for each $l \in\{1,2, \ldots, n\} \backslash\left\{n_{1}, n_{2}, \ldots, n_{s}\right\}, s \geq 2$.

First, we consider case $(4 \mathrm{~A})$. Since $I_{k_{0}}$ is increasing on $\mathbb{R}$, then

$$
\beta_{i_{0}}\left(t_{k_{0}}^{+}\right)=\beta_{i_{0}}\left(t_{k_{0}}\right)+I_{k_{0}}\left(\beta_{i_{0}}\left(t_{k_{0}}\right)\right)<\beta_{j}\left(t_{k_{0}}\right)+I_{k_{0}}\left(\beta_{j}\left(t_{k_{0}}\right)\right)=\beta_{j}\left(t_{k_{0}}^{+}\right), \quad j \neq i_{0} .
$$

Then, there exists $\delta_{0}>0$ small enough such that $\bar{\beta}(t)=\beta_{i_{0}}(t)$ for $t \in\left[t_{k_{0}}-\delta_{0}, t_{k_{0}}+\delta_{0}\right]$ and so $w(t)=x_{0}(t)-\beta_{i_{0}}(t)$ for $t \in\left[t_{k_{0}}-\delta_{0}, t_{k_{0}}+\delta_{0}\right]$. Since $\beta_{i_{0}}(t)$ is a strict upper solution, we 
have

$$
\begin{aligned}
w\left(t_{k_{0}}^{+}\right) & =x_{0}\left(t_{k_{0}}^{+}\right)-\beta_{i_{0}}\left(t_{k_{0}}^{+}\right) \\
& =\left[x_{0}\left(t_{k_{0}}\right)+J_{k_{0}}\left(x_{0}\left(t_{k_{0}}\right)\right)\right]-\left[\beta_{i_{0}}\left(t_{k_{0}}\right)+I_{k_{0}}\left(\beta_{i_{0}}\left(t_{k_{0}}\right)\right)\right] \\
& =\left[x_{0}\left(t_{k_{0}}\right)-\beta_{i_{0}}\left(t_{k_{0}}\right)\right]+\left[J_{k_{0}}\left(x_{0}\left(t_{k_{0}}\right)\right)-I_{k_{0}}\left(\beta_{i_{0}}\left(t_{k_{0}}\right)\right)\right] \\
& =w\left(t_{k_{0}}\right)+\left[I_{k_{0}}\left(\beta_{i_{0}}\left(t_{k_{0}}\right)\right)-I_{k_{0}}\left(\beta_{i_{0}}\left(t_{k_{0}}\right)\right)\right] \\
& =w\left(t_{k_{0}}\right) .
\end{aligned}
$$

Since $w(\tau)<w\left(t_{k_{0}}\right)$ for each $\tau \in\left(t_{k_{0}-1}, t_{k_{0}}\right)$, then we have $w^{\prime}\left(t_{k_{0}}\right) \geq 0$. Similarly, we have $w^{\prime}\left(t_{k_{0}}^{+}\right) \leq 0$. Therefore,

$$
\begin{aligned}
0 & \geq w^{\prime}\left(t_{k_{0}}^{+}\right)=x^{\prime}\left(t_{k_{0}}^{+}\right)-\beta_{i_{0}}^{\prime}\left(t_{k_{0}}^{+}\right) \\
& >\left[x_{0}^{\prime}\left(t_{k_{0}}\right)+\bar{J}_{k_{0}}\left(x_{0}\left(t_{k_{0}}\right)\right)\right]-\left[\beta_{i_{0}}^{\prime}\left(t_{k_{0}}\right)+\bar{I}_{k_{0}}\left(\beta_{i_{0}}\left(t_{k_{0}}\right)\right)\right] \\
& =w^{\prime}\left(t_{k_{0}}\right)+\left[\bar{I}_{k_{0}}\left(\beta_{i_{0}}\left(t_{k_{0}}\right)\right)-\bar{I}_{k_{0}}\left(\beta_{i_{0}}\left(t_{k_{0}}\right)\right)\right]=w^{\prime}\left(t_{k_{0}}\right) \geq 0,
\end{aligned}
$$

which is contradiction.

Now we consider case (4B). Since $I_{k_{0}}$ is increasing, then we have

$$
\bar{\beta}\left(t_{k_{0}}^{+}\right)=\beta_{n_{1}}\left(t_{k_{0}}^{+}\right)=\beta_{n_{2}}\left(t_{k_{0}}^{+}\right)=\cdots=\beta_{n_{s}}\left(t_{k_{0}}^{+}\right)
$$

while $\beta_{l}\left(t_{k_{0}}^{+}\right)>\beta\left(t_{k_{0}}^{+}\right)$for each $l \in\{1,2, \ldots, n\} \backslash\left\{n_{1}, n_{2}, \ldots, n_{s}\right\}$. For case (4B), we have two subcases:

(4Ba) there exists $\delta_{0}>0$ small enough and $i_{0} \in\left\{n_{1}, n_{2}, \ldots, n_{s}\right\}$ such that $\beta_{i_{0}}(t)=\beta(t)$ for $t \in\left[t_{k_{0}}-\delta_{0}, t_{k_{0}}+\delta_{0}\right]$;

$(4 \mathrm{Bb})$ there exists $\delta_{0}>0$ small enough and $i_{0} \neq j_{0}, i_{0}, j_{0} \in\left\{n_{1}, n_{2}, \ldots, n_{s}\right\}$ such that

$$
\bar{\beta}(t)= \begin{cases}\beta_{i_{0}}(t), & t \in\left[t_{k_{0}}-\delta_{0}, t_{k_{0}}\right], \\ \beta_{j_{0}}(t), & t \in\left(t_{k_{0}}, t_{k_{0}}+\delta_{0}\right] .\end{cases}
$$

For case $(4 \mathrm{Ba})$ as in case $(4 \mathrm{~A})$, we can easily obtain a contradiction. For case $(4 \mathrm{Bb})$, we have

$$
w(t)= \begin{cases}x_{0}(t)-\beta_{i_{0}}(t), & t \in\left[t_{k_{0}}-\delta_{0}, t_{k_{0}}\right], \\ x_{0}(t)-\beta_{j_{0}}(t), & t \in\left(t_{k_{0}}, t_{k_{0}}+\delta_{0}\right] .\end{cases}
$$


In the same way as in the proof of case (4A), we see that $w\left(t_{k_{0}}^{+}\right)=w\left(t_{k_{0}}\right), w^{\prime}\left(t_{k_{0}}\right) \geq 0$ and we have $w^{\prime}\left(t_{k_{0}}^{+}\right) \leq 0$. Note that $\beta_{j_{0}}^{\prime}\left(t_{k_{0}}\right) \leq \beta_{i_{0}}^{\prime}\left(t_{k_{0}}\right)$, and we have

$$
\begin{aligned}
0 & \geq w^{\prime}\left(t_{k_{0}}^{+}\right)=x_{0}^{\prime}\left(t_{k_{0}}^{+}\right)-\beta_{j_{0}}^{\prime}\left(t_{k_{0}}^{+}\right) \\
& =\left[x_{0}^{\prime}\left(t_{k_{0}}\right)+\left.\Delta x_{0}^{\prime}\right|_{t=t_{k_{0}}}\right]-\left[\beta_{j_{0}}^{\prime}\left(t_{k_{0}}\right)+\left.\Delta \beta_{j_{0}}^{\prime}\right|_{t=t_{k_{0}}}\right] \\
& >\left[x_{0}^{\prime}\left(t_{k_{0}}\right)-\beta_{j_{0}}^{\prime}\left(t_{k_{0}}\right)\right]+\left[\bar{J}_{k_{0}}\left(x_{0}\left(t_{k_{0}}\right)\right)-\bar{I}_{k_{0}}\left(\beta_{j_{0}}\left(t_{k_{0}}\right)\right)\right] \\
& \geq x_{0}^{\prime}\left(t_{k_{0}}\right)-\beta_{i_{0}}^{\prime}\left(t_{k_{0}}\right)+\left[\bar{I}_{k_{0}}\left(\beta_{j_{0}}\left(t_{k_{0}}\right)\right)-\bar{I}_{k_{0}}\left(\beta_{j_{0}}\left(t_{k_{0}}\right)\right)\right] \\
& =w^{\prime}\left(t_{k_{0}}\right) \geq 0
\end{aligned}
$$

which is a contradiction.

(5) There exists a $k_{0} \in\{1,2, \ldots, m\}$ such that $w\left(t_{k_{0}}^{+}\right)=\sup _{t \in J} w(t)>0$. Without loss of generality, we may assume that $w(\tau)<w\left(t_{k_{0}}^{+}\right)$for each $k \in\{1,2, \ldots, m, m+1\}$ and $\tau \in$ $\left(t_{k-1}, t_{k}\right)$. We have two subcases:

(5A) there exists $i_{0} \in\{1,2, \ldots, n\}$ such that $\beta_{i_{0}}\left(t_{k_{0}}^{+}\right)<\beta_{j}\left(t_{k_{0}}^{+}\right)$for each $j \neq i_{0}$;

(5B) there exists a subset $\left\{n_{1}, n_{2}, \ldots, n_{s}\right\} \subset\{1,2, \ldots, n\}$ such that

$$
\bar{\beta}\left(t_{k_{0}}^{+}\right)=\beta_{n_{1}}\left(t_{k_{0}}^{+}\right)=\beta_{n_{2}}\left(t_{k_{0}}^{+}\right)=\cdots=\beta_{n_{s}}\left(t_{k_{0}}^{+}\right),
$$

while $\beta_{l}\left(t_{k_{0}}^{+}\right)>\bar{\beta}\left(t_{k_{0}}^{+}\right)$for each $l \in\{1,2, \ldots, n\} \backslash\left\{n_{1}, n_{2}, \ldots, n_{s}\right\}, s \geq 2$.

Since $I_{k_{0}}$ is increasing, then for case $(5 \mathrm{~A})$, we have

$$
\beta_{i_{0}}\left(t_{k_{0}}\right)<\beta_{j}\left(t_{k_{0}}\right) \quad\left(j \neq i_{0}\right), \quad x_{0}\left(t_{k_{0}}\right)>\beta_{i_{0}}\left(t_{k_{0}}\right),
$$

and for case (5B), we have $x_{0}\left(t_{k_{0}}\right)>\beta_{i_{0}}\left(t_{k_{0}}\right)$ and

$$
\bar{\beta}\left(t_{k_{0}}\right)=\beta_{n_{1}}\left(t_{k_{0}}\right)=\beta_{n_{2}}\left(t_{k_{0}}\right)=\cdots=\beta_{n_{s}}\left(t_{k_{0}}\right)
$$

while $\beta_{l}\left(t_{k_{0}}\right)>\bar{\beta}\left(t_{k_{0}}\right)$ for each $l \in\{1,2, \ldots, n\} \backslash\left\{n_{1}, n_{2}, \ldots, n_{s}\right\}$. Therefore, we can use the same method as in case (4) to obtain a contradiction.

From the discussions of (1)-(5), we see that $x_{0}(t) \leq \bar{\beta}(t)$ for $t \in J$. Similarly, we can prove that $\bar{\alpha}(t) \leq x_{0}(t)$ for $t \in J$. Thus, (2.33) holds.

Next, we prove that $\bar{\alpha} \prec x_{0} \prec \bar{\beta}$. If the inequality $x_{0} \prec \bar{\beta}$ does not hold, then either there exists $\tau_{0} \in J$ such that $x_{0}\left(\tau_{0}\right)=\bar{\beta}\left(\tau_{0}\right)$ or there exists $k_{0} \in\{1,2, \ldots, m\}$ such that $x_{0}\left(t_{k_{0}}^{+}\right)=$ $\bar{\beta}\left(t_{k_{0}}^{+}\right)$. Set $w(t)=x_{0}(t)-\bar{\beta}(t)$ for $t \in J$. Then, we have either $w\left(\tau_{0}\right)=\sup _{t \in J} w(t)$ or $w\left(t_{k_{0}}^{+}\right)=$ $\sup _{t \in J} w(t)$ for some $k_{0} \in\{1,2, \ldots, m\}$. Essentially the same reasoning as in (1)-(5) above yields a contradiction. Thus, $x_{0} \prec \bar{\beta}$. Similarly, $\bar{\alpha} \prec x_{0}$. Consequently, (2.31) holds.

Step 3. Now, we show (2.32). Suppose not, then we have the following two subcases:

(I) there exists $s_{1} \in J$ such that $\left|x_{0}^{\prime}\left(s_{1}\right)\right| \geq L$;

(II) there exists $k_{0} \in\{1,2, \ldots, m\}$ such that $\left|x_{0}^{\prime}\left(t_{k_{0}}^{+}\right)\right| \geq L$. 
We only consider case (II). A similar argument works for case (I). We may assume without loss of generality that $x_{0}^{\prime}\left(t_{k_{0}}^{+}\right) \geq L$. By the mean-value theorem, there exists $s_{2} \in$ $\left(t_{k_{0}}, t_{k_{0}+1}\right)$ such that

$$
x_{0}^{\prime}\left(s_{2}\right)=\frac{x_{0}\left(t_{k_{0}+1}\right)-x_{0}\left(t_{k_{0}}\right)}{t_{k_{0}+1}-t_{k_{0}}} \leq \frac{\max _{1 \leq i \leq n} \sup _{t \in J} \beta_{i}(t)-\min _{1 \leq i \leq n} \inf _{t \in J} \alpha_{i}(t)}{\mu_{0}}<\lambda<L .
$$

Let $L_{1}$ be such that $L_{0}<L_{1}<L$, then, there exist $s_{3}, s_{4} \in\left(t_{k_{0}}, s_{2}\right]$ such that $s_{3}<s_{4}, x_{0}^{\prime}\left(s_{3}\right)=L_{1}$, $x_{0}^{\prime}\left(s_{4}\right)=\lambda$, and $\lambda \leq x_{0}^{\prime}(s) \leq L_{1}$ for $s \in\left[s_{3}, s_{4}\right]$. Therefore,

$$
\left|x_{0}^{\prime \prime}(s)\right|=\left|h\left(s, x_{0}(s), x_{0}^{\prime}(s)\right)\right|=\left|f\left(s, x_{0}(s), x_{0}^{\prime}(s)\right)\right| \leq \phi\left(x_{0}^{\prime}(s)\right), \quad s \in\left[s_{3}, s_{4}\right] .
$$

Consequently,

$$
\left|\int_{s_{3}}^{s_{4}} \frac{x_{0}^{\prime}(s) x_{0}^{\prime \prime}(s)}{\phi\left(x_{0}^{\prime}(s)\right)} d s\right| \leq \int_{s_{3}}^{s_{4}}\left|x_{0}^{\prime}(s)\right| d s=\int_{s_{3}}^{s_{4}} x_{0}^{\prime}(s) d s=x_{0}\left(s_{4}\right)-x_{0}\left(s_{3}\right)<\lambda .
$$

On the other hand,

$$
\left|\int_{s_{3}}^{s_{4}} \frac{x_{0}^{\prime}(s) x_{0}^{\prime \prime}(s)}{\phi\left(x_{0}^{\prime}(s)\right)} d s\right|=\left|\int_{\lambda}^{L_{1}} \frac{s}{\phi(s)} d s\right| \geq \int_{\lambda}^{N} \frac{s}{\phi(s)} d s>\lambda,
$$

which is a contradiction. Thus, (2.32) holds.

Step 4. From the excision property of Leray-Schauder degree and (2.29), we have

$$
\operatorname{deg}\left(I-A^{*}, \Omega, \theta\right)=\operatorname{deg}\left(I-A^{*}, B(\theta, R), \theta\right)=1 .
$$

From (2.31) and (2.32), we see that $A x=A^{*} x$ for each $x \in \bar{\Omega}$, and so

$$
\operatorname{deg}(I-A, \Omega, \theta)=1
$$

The proof is complete.

Remark 2.9. From the proof of Theorem 2.8, we see that $A$ has no fixed point on $\partial \Omega$.

Theorem 2.10. Suppose that $\left(H_{0}\right),\left(H_{1}\right)$ hold, $u_{1}(t), u_{2}(t)$ are strict lower solutions, $v_{1}(t), v_{0}(t)$ are strict upper solutions, $u_{1} \prec v_{1} \prec v_{0}, u_{2} \prec v_{0}, u_{2}\left(t^{\prime}\right)>v_{1}\left(t^{\prime}\right)$ for some $t^{\prime} \in J$, and $f$ satisfies Nagumo condition with respect to $\left[u_{1}, v_{0}\right]$. Moreover, the strict lower solutions $u_{1}(t), u_{2}(t)$ are well ordered whenever $I_{i_{0}}(x) \neq 0$ or $\bar{I}_{j_{0}}(x) \neq 0$ for some $i_{0}, j_{0} \in\{1,2, \ldots, m\}$ and some $x \in \mathbb{R}$. Then, (1.1) has at least three solutions $x_{1}, x_{2}$, and $x_{3}$, such that

$$
u_{1} \prec x_{1} \prec v_{1}, \quad u_{1} \prec x_{2} \prec v_{0}, \quad u_{2} \prec x_{2} \prec v_{0}, \quad u_{1} \prec x_{3} \prec v_{0},
$$

and $v_{1}\left(s_{1}\right)<x_{3}\left(s_{1}\right), x_{3}\left(s_{2}\right)<u_{2}\left(s_{2}\right)$ for some $s_{1}, s_{2} \in J$. 
Proof. Set $\bar{\alpha}(t)=\max \left\{u_{1}(t), u_{2}(t)\right\}$ for $t \in J$, and $\bar{\alpha}\left(t_{k}^{+}\right)=\max \left\{u_{1}\left(t_{k}^{+}\right), u_{2}\left(t_{k}^{+}\right)\right\}$for each $k \in$ $\{1,2, \ldots, m\}$. From Theorem 2.8, we see that there exist $R>0$ and $L>0$ large enough such that

$$
\begin{aligned}
& \operatorname{deg}\left(I-A, G_{0}, \theta\right)=1, \\
& \operatorname{deg}\left(I-A, G_{1}, \theta\right)=1, \\
& \operatorname{deg}\left(I-A, G_{2}, \theta\right)=1,
\end{aligned}
$$

where $G_{0}=\left\{x \in B(\theta, R) \mid u_{1}<x<v_{0},-L \prec x^{\prime}<L\right\}, G_{1}=\left\{x \in B(\theta, R) \mid u_{1}<x<\right.$ $\left.v_{1},-L<x^{\prime}<L\right\}$, and $G_{2}=\left\{x \in B(\theta, R) \mid \bar{\alpha}<x<v_{0},-L \prec x^{\prime}<L\right\}$. Then, $A$ has fixed points $x_{1} \in G_{1}$ and $x_{2} \in G_{2}$, respectively. From the conditions of Theorem 2.10, we see that $\bar{G}_{1} \cap \bar{G}_{2}=\varnothing$. Let $\omega_{0}(t)$ be a continuous function on $J$ such that its graph passes the points $\left(0,\left(v_{0}(0)+v_{1}(0)\right) / 2\right)$ and $\left(t^{\prime},\left(v_{1}\left(t^{\prime}\right)+u_{1}\left(t^{\prime}\right)\right) / 2\right)$, and satisfies $u_{1} \prec \omega_{0} \prec v_{0}$. By the well-known Weierstrass approximation theorem, there exists $\omega_{1} \in C^{1}[0,1]$ such that

$$
\left|\omega_{1}(t)-\omega_{0}(t)\right|<\min \left\{\frac{v_{1}\left(t^{\prime}\right)-u_{1}\left(t^{\prime}\right)}{4}, \frac{v_{0}(0)-v_{1}(0)}{4}, \frac{1}{4}\left\|\omega_{0}-v_{0}\right\|_{\mathrm{PC}^{\prime}} \frac{1}{4}\left\|\omega_{0}-u_{1}\right\|_{\mathrm{PC}}\right\}, \quad t \in J .
$$

It is easy to see that $\omega_{1} \in G_{0} \backslash\left(\bar{G}_{1} \cup \bar{G}_{2}\right)$, and so $G_{0} \backslash\left(\bar{G}_{1} \cup \bar{G}_{2}\right)$ is a nonempty open set. Note $A$ has no fixed point on $\partial G_{0}, \partial G_{1}$, and $\partial G_{2}$. From (2.58), we have

$$
\operatorname{deg}\left(I-A, G_{0} \backslash\left(\bar{G}_{1} \cup \bar{G}_{2}\right), \theta\right)=\operatorname{deg}\left(I-A, G_{0}, \theta\right)-\operatorname{deg}\left(I-A, G_{1}, \theta\right)-\operatorname{deg}\left(I-A, G_{2}, \theta\right)=-1
$$

Thus, $A$ has at least one fixed point $x_{3} \in G_{0} \backslash\left(\bar{G}_{1} \cup \bar{G}_{2}\right)$. Since $x_{3} \notin \bar{G}_{1} \cup \bar{G}_{2}$, then there exist $s_{1}, s_{2} \in J$ such that $v_{1}\left(s_{1}\right)<x_{3}\left(s_{1}\right)$ and $x_{3}\left(s_{2}\right)<u_{2}\left(s_{2}\right)$. The proof is complete.

Remark 2.11. Theorem 2.10 is a partial generalization of the main results of [16, Theorem 2.2]. Here, we do not need to assume that $u_{2}$ satisfies $u_{1} \leq u_{2} \leq v_{0}$.

Remark 2.12. The position of $u_{1}, u_{2}, v_{1}, v_{0}$ in Theorem 2.10 can be illustrated roughly by Figure 2.

Remark 2.13. The relationship of $u_{1}, u_{2}, v_{1}, v_{0}$ is different from that of [12, Theorems 9 and 10].

Similarly, we have the following result.

Theorem 2.14. Suppose that $\left(H_{0}\right),\left(H_{1}\right)$ hold, $u_{0}, u_{1}$ are strict lower solutions of $(1.1), v_{1}$ and $v_{2}$ are strict upper solutions of (1.1), $u_{0} \prec u_{1} \prec v_{1}, u_{0} \prec v_{2}, v_{2}\left(t^{\prime}\right)<u_{1}\left(t^{\prime}\right)$ for some $t^{\prime} \in J$, and $f$ satisfies Nagumo condition with respect to $\left[u_{0}, v_{1}\right]$. Moreover, the strict upper solutions $v_{1}(t), v_{2}(t)$ are well ordered whenever $I_{i_{0}}(x) \neq 0$ or $\bar{I}_{j_{0}}(x) \neq 0$ for some $i_{0}, j_{0} \in\{1,2, \ldots, m\}$ and some $x \in \mathbb{R}$. 


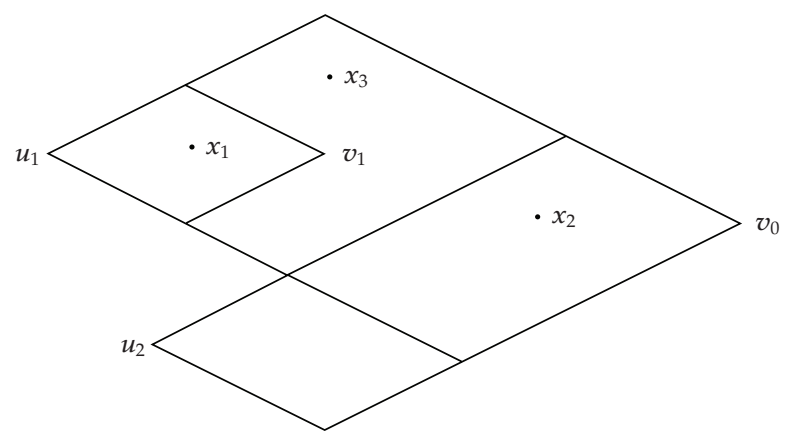

Figure 2: The position of $u_{1}, u_{2}, v_{1}, v_{0}$ in Theorem 2.10.

Then, (1.1) has at least three solutions $x_{1}, x_{2}, x_{3}$ such that

$$
u_{1} \prec x_{1} \prec v_{1}, \quad u_{0} \prec x_{2} \prec v_{1}, \quad u_{0} \prec x_{2} \prec v_{2}, \quad u_{0} \prec x_{3} \prec v_{1},
$$

and $v_{2}\left(s_{1}\right)<x_{3}\left(s_{1}\right), x_{3}\left(s_{2}\right)<u_{1}\left(s_{2}\right)$ for some $s_{1}, s_{2} \in J$.

From Theorems 2.10 and 2.14, we have the following Theorem 2.15.

Theorem 2.15. Suppose that $\left(H_{0}\right),\left(H_{1}\right)$ hold, $u_{0}, u_{1}, u_{2}$ are three strict lower solutions of (1.1), $v_{0}, v_{1}, v_{2}$ are three strict upper solutions of (1.1), $u_{0} \prec u_{1} \prec v_{1} \prec v_{0}, u_{0} \prec u_{2} \prec v_{2} \prec v_{0}$, $u_{2}\left(t^{\prime}\right)>v_{1}\left(t^{\prime}\right), v_{2}\left(t^{\prime \prime}\right)<u_{1}\left(t^{\prime \prime}\right)$ for some $t^{\prime}, t^{\prime \prime} \in J$, and $f$ satisfies Nagumo conditions with respect to $\left[u_{0}, v_{0}\right]$. Moreover, the strict lower solutions $u_{0}, u_{1}, u_{2}$ and the strict upper solutions $v_{0}, v_{1}, v_{2}$ are well ordered whenever $I_{i_{0}}(x) \neq 0$ or $\bar{I}_{j_{0}}(x) \neq 0$ for some $i_{0}, j_{0} \in\{1,2, \ldots, m\}$ and some $x \in \mathbb{R}$. Then, (1.1) has at least eight solutions.

Proof. Now Theorem 2.10 guarantees that (1.1) has at least three solutions $x_{1}, x_{2}, x_{3}$ such that

$$
u_{1} \prec x_{1} \prec v_{1}, \quad u_{1} \prec x_{2} \prec v_{0}, \quad u_{2} \prec x_{2} \prec v_{0}, \quad u_{1} \prec x_{3} \prec v_{0},
$$

and $v_{1}\left(s_{1}\right)<x_{3}\left(s_{1}\right), x_{3}\left(s_{2}\right)<u_{2}\left(s_{2}\right)$ for some $s_{1}, s_{2} \in J$.

Also (1.1) has at least two solutions $x_{4}$ and $x_{5}$ such that

$$
u_{2} \prec x_{4} \prec v_{2}, \quad u_{2} \prec x_{5} \prec v_{0},
$$

and $v_{2}\left(s_{3}\right)<x_{5}\left(s_{3}\right), x_{5}\left(s_{4}\right)<u_{1}\left(s_{4}\right)$.

Now Theorem 2.14 guarantees that (1.1) has at least two solutions $x_{6}, x_{7}$ such that

$$
u_{0} \prec x_{6} \prec v_{2}, \quad u_{0} \prec x_{6} \prec v_{1}, \quad u_{0} \prec x_{7} \prec v_{2},
$$

and $v_{1}\left(s_{5}\right)<x_{7}\left(s_{5}\right), x_{7}\left(s_{6}\right)<u_{2}\left(s_{6}\right)$.

Also (1.1) has at least one solution $x_{8}$ such that $u_{0} \prec x_{8} \prec v_{1}$ and $v_{2}\left(s_{7}\right)<$ $x_{8}\left(s_{7}\right), x_{8}\left(s_{8}\right)<u_{1}\left(s_{8}\right)$ for some $s_{7}, s_{8} \in J$. It is easy to see that $x_{1}, x_{2}, \ldots, x_{8}$ are distinct eight solutions of (1.1). The proof is complete. 


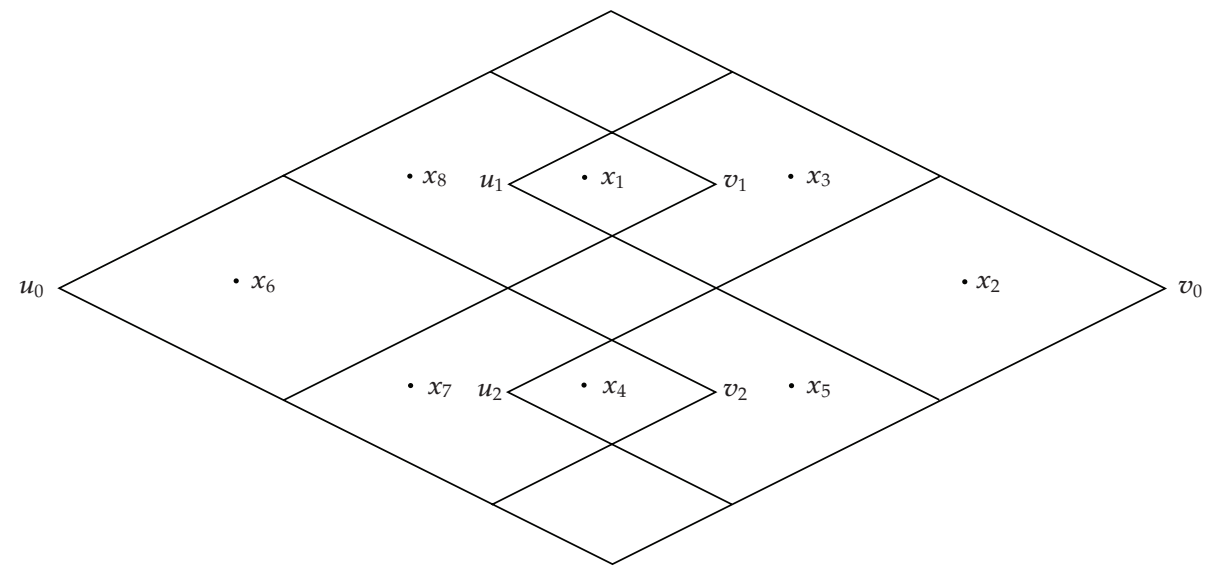

Figure 3: The position of $u_{0}, u_{1}, u_{2}, v_{0}, v_{1}, v_{2}$ in Theorem 2.15.

Remark 2.16. The position of $u_{0}, u_{1}, u_{2}, v_{0}, v_{1}, v_{2}$ in Theorem 2.15 can be illustrated roughly by Figure 3.

\section{Further discussions}

For simplicity, in this section, we will always assume that

$$
I_{k}(x)=\bar{I}_{k}(x)=0, \quad x \in \mathbb{R}, k \in\{1,2, \ldots, m\} .
$$

In this case, (1.1) can be reduced to the following three-point boundary value problem

$$
\begin{gathered}
y^{\prime \prime}+f\left(t, y(t), y^{\prime}(t)\right)=0, \quad t \in(0,1), \\
y(0)=0=y(1)-\alpha y(\eta),
\end{gathered}
$$

where $0<\eta<1$ and $0 \leq \alpha<1$.

In this section, we will use the following assumptions.

$\left(A_{1}\right)$ Suppose that $u_{1}, u_{2}$ are two strict lower solutions, $v_{1}, v_{2}$ are two strict upper solutions of (1.1), $u_{1} \prec v_{1}, u_{2} \prec v_{2}$, and $u_{2}\left(s_{1}\right)>v_{1}\left(s_{1}\right), u_{1}\left(s_{2}\right)>v_{2}\left(s_{2}\right)$ for some $s_{1}, s_{2} \in J$.

Recently, this multipoint boundary value problem has been studied by many authors, see $[16,17,19-21]$ and the references therein. The goal of this section is to prove some multiplicity results for (3.2) using the condition of two pairs of strict upper and lower solutions. As we can see from [13], some bounding condition on the nonlinear term is needed. Instead of the space $\operatorname{PC}^{1}[J, \mathbb{R}]$, in this section we will use the space $C^{1}(J)$. First, we have the following theorem.

Theorem 3.1. Suppose that $\left(A_{1}\right)$ holds, and

$$
|f(t, x, y)|<D_{0}, \quad(t, x, y) \in J \times \mathbb{R}^{2}
$$

for some $D_{0}>0$. Then, (3.2) has at least eight solutions. 
Proof. First, we show that there exist strict lower and upper solutions $u_{0}, v_{0}$ such that

$$
u_{0} \prec u_{i} \prec v_{i} \prec v_{0}, \quad i=1,2 .
$$

Let $a_{0}>\left\|u_{1}\right\|_{C}+\left\|u_{2}\right\|_{C}+\left\|v_{1}\right\|_{C}+\left\|v_{2}\right\|_{C}+1$. Now, we consider the following boundary value problem:

$$
\begin{gathered}
v_{0}^{\prime \prime}(t)+D_{0}=0, \quad t \in(0,1), \\
v_{0}(0)=a_{0}=v_{0}(1)-\alpha v_{0}(\eta) .
\end{gathered}
$$

Let

$$
\begin{gathered}
g_{1}(t)=\frac{1-\alpha \eta+\alpha t}{1-\alpha \eta}, \quad t \in J, \\
g_{2}(t)=\frac{t}{2(1-\alpha \eta)}-\frac{\alpha \eta^{2} t}{2(1-\alpha \eta)}-\frac{1}{2} t^{2}, \quad t \in J .
\end{gathered}
$$

By Lemma 2.7, we have

$$
\begin{aligned}
v_{0}(t) & =g_{1}(t) a_{0}+\frac{D_{0} t}{1-\alpha \eta} \int_{0}^{1}(1-s) d s-\frac{\alpha D_{0} t}{1-\alpha \eta} \int_{0}^{\eta}(\eta-s) d s-D_{0} \int_{0}^{t}(t-s) d s \\
& =g_{1}(t) a_{0}+g_{2}(t) D_{0}, \quad t \in J .
\end{aligned}
$$

It is easy to see that $g_{1}(t) \geq 1$ and $g_{2}(t) \geq 0$ for each $t \in J$. Thus, $v_{0}(t) \geq a_{0}$ for each $t \in J$, and therefore, $u_{i} \prec v_{0}, v_{i} \prec v_{0}$ for $i=1$,2. On the other hand, from (3.5), it is easy to see that $v_{0}$ is a strict upper solution of (1.1). Similarly, we can show the existence of $u_{0}$. Then, by Theorem 2.15, the conclusion holds.

Remark 3.2. Obviously, the condition (3.3) is restrictive. In the following, we will make use of a weaker condition. We study the multiplicity of solutions of (3.2) under a NagumoKnobloch-Schmitt condition. For this kind of bounding condition, the reader is referred to [13].

Theorem 3.3. Suppose $\left(A_{1}\right)$ holds, and there exists function $\beta_{1}, \beta_{2} \in C^{1}(J), \beta_{1} \leq \beta_{2}$ such that

$$
\begin{gathered}
\beta_{1}(t) \leq u_{i}^{\prime}(t) \leq \beta_{2}(t), \quad \beta_{1}(t) \leq v_{i}^{\prime}(t) \leq \beta_{2}(t), \quad t \in J, \\
-f\left(t, x, \beta_{1}(t)\right)<\beta_{1}^{\prime}(t), \quad-f\left(t, x, \beta_{2}(t)\right)>\beta_{2}^{\prime}(t), \quad(t, x) \in J \times\left[s_{2}, s_{1}\right], \\
\beta_{1}(1) \leq-\frac{2}{1-\alpha \eta} D_{0} \leq \frac{2}{1-\alpha \eta} D_{0} \leq \beta_{2}(1),
\end{gathered}
$$


where $D_{0}=\max _{(t, x, y) \in J \times\left[s_{2}, s_{1}\right] \times \mathbb{R}}|f(t, y, \phi(t, y))|, \gamma=\left\|u_{1}\right\|_{C}+\left\|v_{1}\right\|_{C}+\left\|u_{2}\right\|_{C}+\left\|v_{2}\right\|_{C}$,

$$
\begin{gathered}
s_{2}=-\int_{0}^{1}\left|\beta_{1}(t)\right| d t-\gamma, \quad s_{1}=\int_{0}^{1}\left|\beta_{2}(t)\right| d t+\gamma, \\
\phi(t, y)= \begin{cases}\beta_{2}(t), & y>\beta_{2}(t), \\
y, & \beta_{1}(t) \leq y \leq \beta_{2}(t), \\
\beta_{1}(t), & y<\beta_{1}(t) .\end{cases}
\end{gathered}
$$

Then, (3.2) has at least eight solutions.

Proof. Let $\rho(x)=\max \left\{s_{2}, \min \left\{x, s_{1}\right\}\right\}$ for each $x \in \mathbb{R}$, and

$$
f^{*}(t, x, y)=f(t, \rho(x), \phi(t, y)), \quad(t, x, y) \in J \times \mathbb{R}^{2} .
$$

Now, we consider the following boundary value problem:

$$
\begin{gathered}
y^{\prime \prime}(t)+f^{*}\left(t, y(t), y^{\prime}(t)\right)=0, \quad t \in(0,1), \\
y(0)=0=y(1)-\alpha y(\eta) .
\end{gathered}
$$

From $\left(A_{1}\right)$ and (3.8), we see that $u_{1}, u_{2}$ are strict lower solutions of (3.13), and $v_{1}$ and $v_{2}$ are two strict upper solutions of (3.13). By Theorem 3.1, (3.13) has at least eight solutions $x_{1}, x_{2}, \ldots, x_{8}$. We need only to show that $x_{1}, x_{2}, \ldots, x_{8}$ are solutions of (3.2). We claim that

$$
\beta_{1}(t) \leq x_{i}^{\prime}(t) \leq \beta_{2}(t), \quad t \in J, i=1,2, \ldots, 8
$$

We only show that $x_{1}^{\prime}(t) \leq \beta_{2}(t)$ for $t \in J$. If $x_{1}^{\prime}\left(t^{\prime}\right)>\beta_{2}\left(t^{\prime}\right)$ for some $t^{\prime} \in J$, then $\max _{t \in J} z(t)=$ $z\left(t_{0}\right)>0$ for some $t_{0} \in J$, where $z(t)=x_{1}^{\prime}(t)-\beta_{2}(t)$ for $t \in J$. If $t_{0} \in[0,1)$, then $z^{\prime}\left(t_{0}\right) \leq 0$, and so

$$
\begin{aligned}
0 & \geq z^{\prime}\left(t_{0}\right)=x_{1}^{\prime \prime}\left(t_{0}\right)-\beta_{2}^{\prime}\left(t_{0}\right)=-f^{*}\left(t_{0}, x_{1}\left(t_{0}\right), x_{1}^{\prime}\left(t_{0}\right)\right)-\beta_{2}^{\prime}\left(t_{0}\right) \\
& =-f\left(t_{0}, \rho\left(x_{1}\left(t_{0}\right)\right), \phi\left(t_{0}, x_{1}^{\prime}\left(t_{0}\right)\right)-\beta_{2}^{\prime}\left(t_{0}\right)\right. \\
& =-f\left(t_{0}, \rho\left(x_{1}\left(t_{0}\right)\right), \beta_{2}\left(t_{0}\right)\right)-\beta_{2}^{\prime}\left(t_{0}\right),
\end{aligned}
$$

which contradicts (3.9).

From Lemma 2.6, we have

$$
\begin{aligned}
x_{1}(t)= & \frac{t}{1-\alpha \eta} \int_{0}^{1}(1-s) f^{*}\left(s, x(s), x^{\prime}(s)\right) d s-\frac{\alpha t}{1-\alpha \eta} \int_{0}^{\eta}(\eta-s) f^{*}\left(s, x(s), x_{1}^{\prime}(s)\right) d s \\
& -\int_{0}^{t}(t-s) f^{*}\left(s, x(s), x_{1}^{\prime}(s)\right) d s,
\end{aligned}
$$


and so

$$
x_{1}^{\prime}(t) \leq\left|x_{1}^{\prime}(t)\right| \leq\left[\frac{1}{1-\alpha \eta} \int_{0}^{1}(1-s) d s+\frac{\alpha}{1-\alpha \eta} \int_{0}^{\eta}(\eta-s) d s+1\right] \leq \frac{2}{1-\alpha \eta} D_{0} \leq \beta_{2}(1) .
$$

This implies that $t_{0} \neq 1$. Therefore, (3.14) holds. Integrating (3.14), we have

$$
s_{2} \leq-\int_{0}^{1}\left|\beta_{1}(t)\right| d t \leq x_{1}(t) \leq \int_{0}^{1}\left|\beta_{2}(t)\right| d t \leq s_{1}, \quad t \in J .
$$

From (3.13)-(3.18), we see that $x_{1}, x_{2}, \ldots, x_{8}$ are eight solutions of (3.2). The proof is complete.

Remark 3.4. We also can replace (3.3) by other bounding conditions, see [13].

Remark 3.5. To end this paper, we point out that the results of this paper can be applied to study the multiplicity of radial solutions of elliptic differential equation in an annulus with impulses at some radii.

\section{Acknowledgments}

This paper is supported by Natural Science Foundation of Jiangsu Education Committee (04KJB110138) and China Postdoctoral Science Foundation (2005037712).

\section{References}

[1] D. Guo, "Periodic boundary value problems for second order impulsive integro-differential equations in Banach spaces," Nonlinear Analysis: Theory, Methods \& Applications, vol. 28, no. 6, pp. 983-997, 1997.

[2] V. Lakshmikantham, D. D. Bănov, and P. S. Simeonov, Theory of Impulsive Differential Equations, vol. 6 of Series in Modern Applied Mathematics, World Scientific, Teaneck, NJ, USA, 1989.

[3] D. Guo, "Multiple positive solutions of a boundary value problem for $n$ th-order impulsive integrodifferential equations in a Banach space," Nonlinear Analysis: Theory, Methods \& Applications, vol. 56, no. 7, pp. 985-1006, 2004.

[4] L. H. Erbe and X. Liu, "Quasi-solutions of nonlinear impulsive equations in abstract cones," Applicable Analysis, vol. 34, no. 3-4, pp. 231-250, 1989.

[5] X. Liu, "Nonlinear boundary value problems for first order impulsive integro-differential equations," Applicable Analysis, vol. 36, no. 1-2, pp. 119-130, 1990.

[6] E. Picard, "Sur l'application des méthodes d'approximations successives à l' étude de certaines équations différentielles ordinaries," Journal de Mathématiques Pures et Appliquées, vol. 9, pp. 217-271, 1893.

[7] C. De Coster and P. Habets, "An overview of the method of lower and upper solutions for ODEs," in Nonlinear Analysis and Its Applications to Differential Equations, vol. 43, pp. 3-22, Birkhăuser, Boston, Mass, USA, 2001.

[8] I. Rachůnková and M. Tvrdý, "Non-ordered lower and upper functions in second order impulsive periodic problems," Dynamics of Continuous, Discrete \& Impulsive Systems. Series A, vol. 12, no. 3-4, pp. $397-415,2005$.

[9] H. Amann, A. Ambrosetti, and G. Mancini, "Elliptic equations with noninvertible Fredholm linear part and bounded nonlinearities," Mathematische Zeitschrift, vol. 158, no. 2, pp. 179-194, 1978.

[10] C. De Coster and M. Henrard, "Existence and localization of solution for second order elliptic BVP in presence of lower and upper solutions without any order," Journal of Differential Equations, vol. 145, no. 2, pp. 420-452, 1998.

[11] I. Rachünková and M. Tvrdý, "Periodic problems with $\varphi$-Laplacian involving non-ordered lower and upper functions," Fixed Point Theory, vol. 6, no. 1, pp. 99-112, 2005. 
[12] I. Rachůnková, "Upper and lower solutions and topological degree," Journal of Mathematical Analysis and Applications, vol. 234, no. 1, pp. 311-327, 1999.

[13] I. Rachůnková, "Upper and lower solutions and multiplicity results," Journal of Mathematical Analysis and Applications, vol. 246, no. 2, pp. 446-464, 2000.

[14] P. Habets and P. Omari, "Existence and localization of solutions of second order elliptic problems using lower and upper solutions in the reversed order," Topological Methods in Nonlinear Analysis, vol. 8 , no. 1 , pp. 25-56, 1996.

[15] X. Xu, D. O'Regan, and J. Sun, "Multiplicity results for three-point boundary value problems with a non-well-ordered upper and lower solution condition," Mathematical and Computer Modelling, vol. 45, no. 1-2, pp. 189-200, 2007.

[16] R. A. Khan and J. R. L. Webb, “Existence of at least three solutions of a second-order three-point boundary value problem," Nonlinear Analysis: Theory, Methods E Applications, vol. 64, no. 6, pp. 13561366, 2006.

[17] X. Xian, "Three solutions for three-point boundary value problems," Nonlinear Analysis: Theory, Methods \& Applications, vol. 62, no. 6, pp. 1053-1066, 2005.

[18] D. Guo, J. Sun, and Z. Liu, The Funtional Method for Nonlinear Ordinary Differential Equations, Shandong Science and Technology Press, Jinan, China, 1995.

[19] B. Liu, "Positive solutions of second-order three-point boundary value problems with change of sign," Computers \& Mathematics with Applications, vol. 47, no. 8-9, pp. 1351-1361, 2004.

[20] C. P. Gupta and S. I. Trofimchuk, "Existence of a solution of a three-point boundary value problem and the spectral radius of a related linear operator," Nonlinear Analysis: Theory, Methods E Applications, vol. 34, no. 4, pp. 489-507, 1998.

[21] R. Ma and N. Castaneda, "Existence of solutions of nonlinear $m$-point boundary-value problems," Journal of Mathematical Analysis and Applications, vol. 256, no. 2, pp. 556-567, 2001. 